\title{
Community structure and fish assemblage at Marsa Abu Dabab, Red Sea, Egypt.
}

\author{
Magdy A. Alwany \\ Department of Marine Science, Faculty of Science, Suez Canal \\ University, Ismailia, Egypt \\ (e-mail magdy.elalwany@yahoo.com)
}

\begin{abstract}
The fish assemblages of different habitats at Marsa Abu Dabab (north Marsa Alam, Red Sea) were examined by visual census techniques. Fish communities were estimated for each of the investigated habitats; seagrasses and coral reefs which have divided into three sectors; sector A (at north) and C (at south) represent coral reef habitats, whereas sector B (in the middle) represents seagrass habitat. A total of 172 fish species representing 94 genera were recorded at Marsa Abu Dabab. The coral reef habitat had the highest number of species (162 species), while the seagrass habitat had the lowest (90 species). The highest average abundance was recorded at sector $\mathrm{C}$ in the reef wall habitat (1119 fish/1000 $\mathrm{m}^{3}$ ), with the lowest value at sector B in the seagrass habitat $\left(240 \mathrm{fish} / 1000 \mathrm{~m}^{3}\right)$. Some fish species were restricted to seagrass or coral reefs, while others were widespread along the gradient. Regarding to fish distribution patterns, five families showed increasing in coral reef habitat (Pomacentridae, Labridae, Acanthuridae, Chaetodontidae and Serranidae), while other three families were abundant in seagrass beds (Siganidae, Sparidae and Mullidae). The five families in coral reef habitats showed in high abundance on reef wall (RW) more than reef flat (RF), except Acanthuridae.
\end{abstract}

Key words: Marsa Abu Dabab, coral reefs, diversity, fishes, Egypt, Red Sea, seagrass

\section{INTRODUCTION}

Coral reefs rank among the most productive, diverse, and complex ecosystems on earth (Birkeland, 1997). Also, seagrass meadows have extremely high primary and secondary productivity and support a great abundance and diversity of fish (Gillanders, 2006). Human pressures on coral reefs are escalating at unprecedented rates and spatial scales (Hughes et al., 2003), and reefs are declining globally at an alarming rate (Wilkinson, 2004). Many factors contribute to the worldwide degradation of coral reefs and seagrasses. Most prominent amongst the multiple human threats are destructive fishing practices, impacts of tourism, excess nutrient inputs, global warming, and harvesting of corals as building material (Bellwood et al., 2004; Birkeland, 2004). Coastal bays (Marsa) are subject to factors that reflect the interaction between land and sea. Their ecological functions are more complicated and fragile than those of 
the open waters because of the effect of many human activities and the result of land-source pollution.

Roberts et al. (2002) and Mora et al. (2003) considered the Red Sea as a multitaxa center of endemism and fish center of endemism, respectively. Corals, fishes and other organisms of this area create distinct assemblages (Ormond and Edwards, 1987). In the Red Sea region, the status of coral reef and seagrass ecosystems is generally good, with coral cover averaging 16-67 \% and coral bleaching as yet having little effect. Nevertheless, coral and seagrass cover has declined in some areas by over $30 \%$ due to anthropogenic influences (Kotb et al., 2004).

In order to preserve coral reefs by delimiting marine reserve and establishing effective protection measures, it is essential to understand how biomes change on space and time scale (Plotkin and Muller-Landau, 2002; Pandolifi et al., 2003). But we are just beginning to learn more about spatial characteristics of coral and seagrass diversity within and among reef systems and the mechanisms that maintain this diversity (Karlson and Cornell, 1998; Hughes et al., 2002). Therefore, more research is needed to monitor and assess coral reef ecosystems, which will be conducive to profound understanding of the ecological integrity, further amendment of the protection strategy and improvement of management so as to make coral reef and seagrass maintain their high biodiversity and play significant ecological functions. Thus, the objectives of this study were to describe and assess fish communities inside the coral reef and seagrass ecosystems of Marsa Abu Dabab in the northern Red Sea. This inventory will serve as baseline data for future comparisons between different areas or after intensive tourism activities.

\section{MATERIALS AND METHODS}

In order to assess the status of fish communities of Marsa Abu Dabab, long field trip was carried out, for investigation the north to south Marsa Abu Dabab fringing reefs in October 2010. Each transect was almost vertical to the shore and each transect was surveyed using both snorkeling and SCUBA diving. Fish species were identified according to Randall (1983).

\section{Study area}

The Red Sea contains some of the world's most unique and diverse marine and coastal habitats. Marsa Abu Dabab or Abu Dabab bay (25 $31^{\prime} \mathrm{N}, 34^{\circ} 45^{\prime} \mathrm{E}$ ) is located north of Marsa Alam City and considered as a home of dugong (Dugong dugon) sightings in the Red Sea and has become one of the most popular diving spots in Egypt's southern Red Sea coast (Fig. 1). The sheltered and sandy bay of Abu Dabab offers many ideal conditions as a dugong habitat, including one of the largest patches of seagrasses in the region. The bay is also well known as a nesting site for sea turtles (green turtle: Chelonia mydas).

The reef flat extended from the shoreline to the reef edge with a length varied between 40-90 $\mathrm{m}$ in north sector (sector A) and 50-80 m in south sector (sector 
C). On the average, the living cover on the reef flat increased seaward to the reef edge with the highest cover in the reef wall. The middle area of Abu Dabab is sandy sector (sector B) covered by seagrass beds. In the present study, three sectors were studied along Marsa Abu Dabab, north, sandy (middle) and south sectors as shown in Figure 1.

\section{Research Methods}

The underwater survey was conducted using line transect, quadrate and fish census survey techniques followed the international standards of ASEAN (English et al., 1997). The fish communities in shallow water habitats inside Abu Dabab reef and Bay were examined by using visual censuses, which is the most non-destructive method to quantify fish abundance (Sale, 1980). The species were counted visually along $100 \mathrm{~m}$ long, $10 \mathrm{~m}$ wide and $1 \mathrm{~m}$ high transects $\left(100 \times 10 \times 1=1000 \mathrm{~m}^{3}\right)$ laid parallel to the shoreline (three transects in each habitat or sector with three replicates for each transect). Fish communities were estimated for each different bottom habitat, i.e. inner, middle and outer reef flat and reef slope until $10 \mathrm{~m}$. Also, fish communities were estimated in seagrass habitats inside Abu Dabab Bay.

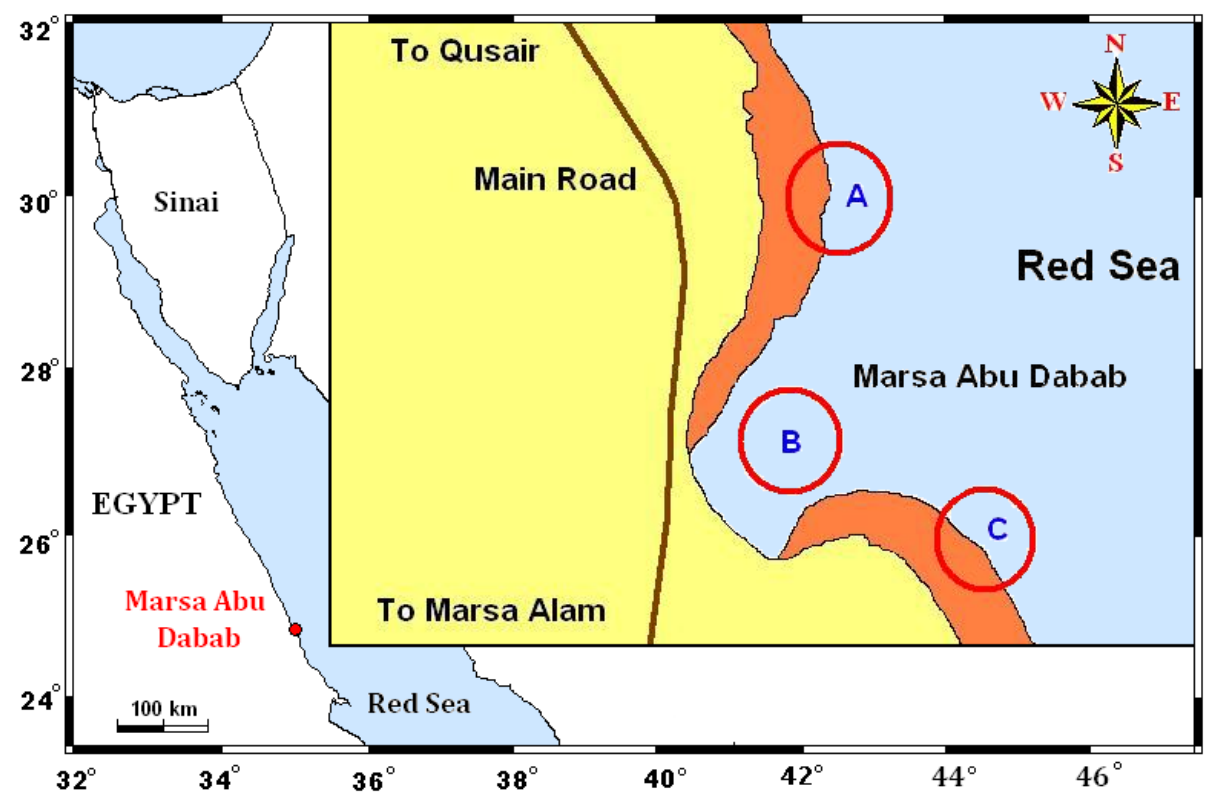

Fig. 1: Map of the northern Red Sea shown location of Marsa Abu Dabab, and three studied sectors A, B and C.

\section{Data analysis}

The data were analysed statistically using the software packages PRIMER (V 5.0) and SPSS (V 15). Species richness was expressed by considering the number of species (D), and species diversity and homogeneity were determined 
using the Shannon-Wiener diversity index $\left(\mathrm{H}^{\prime}\right)$ and the evenness index ( $\left.\mathrm{J}^{\prime}\right)$ (Pielou, 1969). One-way ANOVA was carried out with SPSS program. When necessary, the data were square root transformed to produce normality and homogeneity of variance.

\section{RESULTS}

\section{General description of the three sectors}

For studying coral reef and seagrass communities, a bathymetric map is important as a base map. As it indicates the depths at which the corals occur, it can give an insight into the ecology of reef and seagrass systems. Bathymetric map of Marsa Abu Dabab is illustrated in Figure (2), whereas coral reefs found in north and south of Marsa Abu Dabab. Seagrass beds are found in the middle of sandy area with gradient depths (Fig. 2).

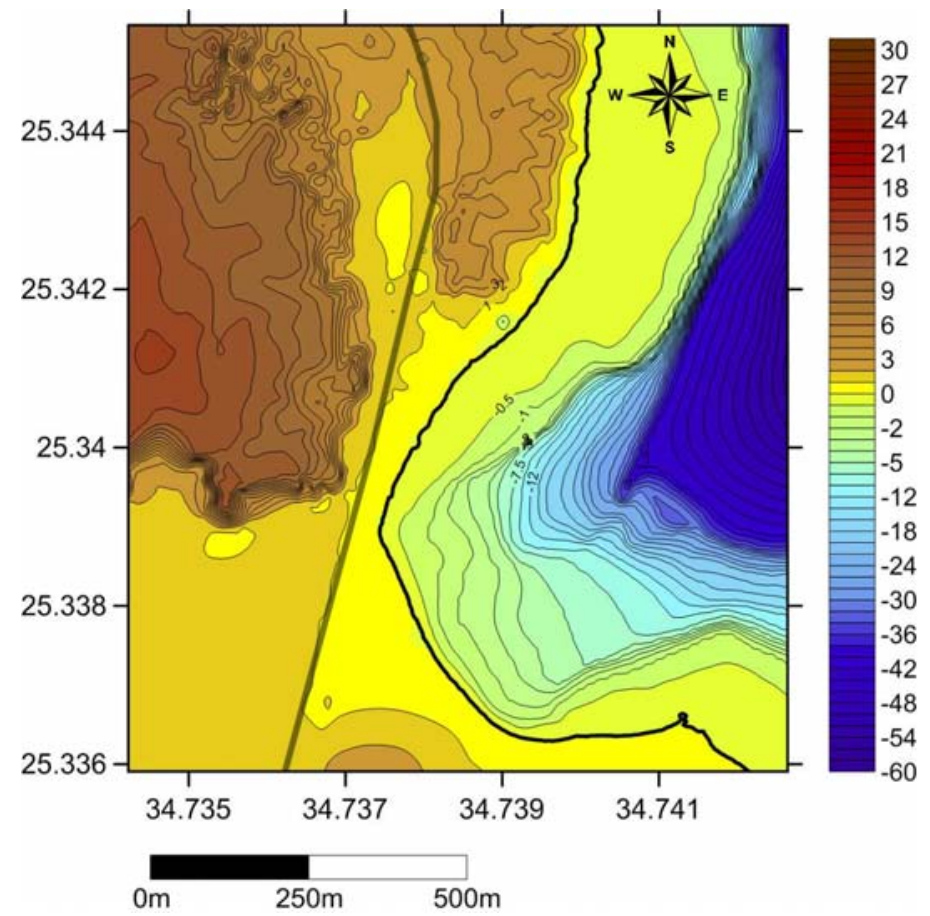

Fig. 2: Bathrymetric map of Marsa Abu Dabab showing gradient of depths.

North Sector (A): The inner reef flat is characterized by high non-living substrate (about $90 \%$ ), whereas at the middle reef, the contribution of living materials is mainly flourished by algae. On the other hand, the outer reef flat and slope are characterized by well-developed coral communities. Along north sector, the coral communities comprised many soft and hard corals. Soft coral colonies are mainly Labophytum, Nephthyia, Sinularia, Cladiella and 
Sarcophyton, whereas, the hard corals are represented on reefs by Acropora, Pocillopora, Favites, Echinopora, Galaxea, Favia, Porites, Pavona, Stylophora and Montipora. Non-true corals are represented by Millepora and Tubipora. Some invertebrates, such as mollusks, echinoderms and crustaceans, were recorded. Mollusks were represented by bivalves (Tridacna) and gastropods (Acanthopleura, Nerita, and Conus); by Echinometra, Macrophiothrix and Diadema, and by different species of crabs and shrimps. Many genera of turf algae are dominated such as brown algae (Dictyota, Cystoseira, Padina and Pocockiella) and red algae (Laurencia, Polysiphonia, Jania, Hypnea and Herposiphonia).

Sandy (middle) Sector (B): this sector is characterised by sandy bottom covered by seagrass beds which comprised very special marine communities. The seagrass community is represented by four species; Halophila stipulacea, $H$. ovalis, Halodule uninervis and Syrngodium isotfolium. Halodule uninervis occurred in moderately covers on the reef flat, while $H$. stipulacea was recorded in high covers inside the bay and at the sandy bottom near the reefs until 30-50 $\mathrm{m}$ depth. The seagrass community comprised many organisms such as gastropods (Strombus tricornis), sea cucumber (Holothurian atra), crabs and sponges. In addition, this community comprised two endangered species dugong (Dugong dugon) and green turtle (Chelonia mydas).

South Sector (C): The inner reef flat is characterized by high non-living substrate, whereas at the middle reef, the contribution of living materials is mainly flourished by algae, where the outer reef flat and slope are characterized by well-developed coral communities. Soft coral colonies are mainly Labophytum, Nephthyia, Sinularia, Cladiella and Sarcophyton, whereas, the hard corals are represented on reefs by Acropora, Pocillopora, Favites, Favia, Porites, Pavona, Stylophora and Montipora. Non-true corals are represented by Millepora and Tubipora. Some invertebrates, such as mollusks, echinoderms and crustaceans, were recorded. They were represented by bivalves (e.g. Tridacna sp.) and gastropods (Planaxis and Conus); by Echinometra, Holothuria, Ophiocoma and Diadema, and by different species of crabs and shrimps, respectively. Many genera of turf algae are dominated (Caulerpa, Dictyota, Padina, Pocockiella, Laurencia, Polysiphonia, Jania, Hypnea and Herposiphonia).

\section{General distribution and abundance of fish species}

The species recorded in each habitat at Marsa Abu Dabab are listed in Table 1. A total of 172 fish species representing 94 genera were counted. Coral reef habitat had the highest number of species (162 species), while the seagrass habitat had the lowest number (90 species). 
Table 1: Abundance (no. of individuals $/ 1000 \mathrm{~m}^{3}$ ) of the different fish species found at each sectors in Marsa Abu Dabab, Red Sea (RF: reef flat, RW reef wall).

\begin{tabular}{|c|c|c|c|c|c|}
\hline \multirow[t]{2}{*}{ Fish species } & \multicolumn{2}{|c|}{ Sector A } & \multirow{2}{*}{$\begin{array}{l}\text { Sector B } \\
\text { Seagrass }\end{array}$} & \multicolumn{2}{|c|}{ Sector C } \\
\hline & RF & RW & & RF & RW \\
\hline \multicolumn{6}{|l|}{ Dasyatididae } \\
\hline Taeniura lymma & 0 & $3.7 \pm 2.1$ & $1.3 \pm 0.6$ & 0 & $3.0 \pm 1.7$ \\
\hline \multicolumn{6}{|l|}{ Synodontidae } \\
\hline Synodus variegatus & $0.3 \pm 0.6$ & $4.3 \pm 1.5$ & $1.7 \pm 0.6$ & $0.7 \pm 0.6$ & $4.7 \pm 1.2$ \\
\hline Saurida gracilis & $0.7 \pm 1.2$ & $6.0 \pm 1.0$ & 0 & $1.3 \pm 1.5$ & $6.7 \pm 0.6$ \\
\hline \multicolumn{6}{|l|}{ Muraenidae } \\
\hline Siderea grisea & $0.3 \pm 0.6$ & 0 & 0 & $1.0 \pm 1.7$ & 0 \\
\hline Gymnothorax flavimarginatus & 0 & $1.3 \pm 0.6$ & 0 & 0 & $1.7 \pm 0.6$ \\
\hline Gymnothorax nudivomer & 0 & $0.3 \pm 0.6$ & 0 & 0 & $0.7 \pm 0.6$ \\
\hline \multicolumn{6}{|l|}{ Belonidae } \\
\hline Tylosurus choram & 0 & $5.7 \pm 0.6$ & 0 & $0.7 \pm 1.2$ & $5.7 \pm 1.5$ \\
\hline \multicolumn{6}{|l|}{ Hemiramphidae } \\
\hline Hyporhamphus far & 0 & $3.3 \pm 3.1$ & $4.7 \pm 1.5$ & 0 & $3.0 \pm 2.6$ \\
\hline Hyporhamphus gambrur & 0 & $2.0 \pm 0$ & $3.0 \pm 1.7$ & 0 & $1.7 \pm 0.6$ \\
\hline \multicolumn{6}{|l|}{ Atherinidae } \\
\hline Atherinomorus lacunosus & 0 & 0 & $3.7 \pm 2.1$ & 0 & $1.3 \pm 1.2$ \\
\hline \multicolumn{6}{|l|}{ Fistulariidae } \\
\hline Fistularia commersonii & 0 & $2.3 \pm 0.6$ & $0.7 \pm 1.2$ & 0 & $2.3 \pm 0.6$ \\
\hline \multicolumn{6}{|l|}{ Syngnathidae } \\
\hline Hippocampus histrix & $0.3 \pm 0.6$ & $0.3 \pm 0.6$ & $0.7 \pm 1.2$ & $1.0 \pm 1.0$ & $1.3 \pm 1.5$ \\
\hline Trachyrhamphus bicoarctatus & $0.7 \pm 1.2$ & 0 & $2.7 \pm 0.6$ & $1.3 \pm 0.6$ & $0.7 \pm 1.2$ \\
\hline Corythoichthys schultzi & $18.0 \pm 3.6$ & $1.3 \pm 0.6$ & $1.3 \pm 0.6$ & $9.7 \pm 2.1$ & $7.0 \pm 2.6$ \\
\hline \multicolumn{6}{|l|}{ Antennariidae } \\
\hline Antennarius coccineus & $0.7 \pm 0.6$ & $0.3 \pm 0.6$ & $1.3 \pm 1.5$ & $0.3 \pm 0.6$ & $0.3 \pm 0.6$ \\
\hline \multicolumn{6}{|l|}{ Anomalopidae } \\
\hline Photoblepharon palpebratus & 0 & $1.7 \pm 2.1$ & 0 & 0 & $0.7 \pm 0.6$ \\
\hline \multicolumn{6}{|l|}{ Holocentridae } \\
\hline Adioryx spinifer & 0 & $3.3 \pm 1.5$ & 0 & 0 & $3.7 \pm 1.5$ \\
\hline Adioryx diadema & 0 & $11.3 \pm 1.5$ & 0 & $1.3 \pm 1.2$ & $11.0 \pm 1.7$ \\
\hline Adioryx ruber & 0 & $3.3 \pm 0.6$ & 0 & 0 & $2.3 \pm 2.1$ \\
\hline Myripristis murdjan & 0 & $1.3 \pm 1.2$ & 0 & 0 & $1.7 \pm 1.5$ \\
\hline Flammeo sammara & 0 & $5.0 \pm 1.0$ & 0 & $0.7 \pm 1.2$ & $5.3 \pm 1.5$ \\
\hline \multicolumn{6}{|l|}{ Serranidae } \\
\hline Cephalopholis argus & $0.7 \pm 0.6$ & $3.3 \pm 1.5$ & 0 & $2.3 \pm 2.3$ & $4.3 \pm 1.2$ \\
\hline Cephalopholis miniata & 0 & $4.3 \pm 2.1$ & 0 & 0 & $4.3 \pm 2.1$ \\
\hline Cephalopholis oligosticta & 0 & $1.0 \pm 1.0$ & 0 & 0 & $1.7 \pm 0.6$ \\
\hline Epinephelus fasciatus & $0.7 \pm 0.6$ & $2.3 \pm 1.2$ & $0.3 \pm 0.6$ & $1.7 \pm 1.2$ & $1.3 \pm 1.5$ \\
\hline Epinephelus malabaricus & 0 & $1.3 \pm 0.6$ & 0 & 0 & $2.0 \pm 1.0$ \\
\hline Epinephelus areolatus & 0 & $3.7 \pm 0.6$ & 0 & 0 & $4.3 \pm 1.5$ \\
\hline Epinephelus chlorostigma & 0 & $1.3 \pm 1.2$ & 0 & 0 & $1.7 \pm 0.6$ \\
\hline Epinephelus stoliczkae & 0 & $3.7 \pm 1.2$ & 0 & 0 & $4.0 \pm 1.7$ \\
\hline
\end{tabular}


Table 1: continued.

\begin{tabular}{|c|c|c|c|c|c|}
\hline \multirow{2}{*}{ Fish species } & \multicolumn{2}{|c|}{ Sector A } & \multirow{2}{*}{$\begin{array}{l}\text { Sector B } \\
\text { Seagrass }\end{array}$} & \multicolumn{2}{|c|}{ Sector C } \\
\hline & RF & RW & & $\mathrm{RF}$ & RW \\
\hline Variola louti & 0 & $1.7 \pm 0.6$ & $0.7 \pm 1.2$ & 0 & $2.3 \pm 1.5$ \\
\hline Anthias squamipinnis & $6.7 \pm 11.5$ & $183.3 \pm 15.3$ & 0 & $10.7 \pm 8.6$ & $146.7 \pm 30.6$ \\
\hline Anthias taeniatus & $1.3 \pm 2.3$ & $23.0 \pm 1.7$ & 0 & $2.7 \pm 2.3$ & $25.3 \pm 3.5$ \\
\hline \multicolumn{6}{|l|}{ Priacanthidae } \\
\hline Priacanthus hamrur & 0 & $1.7 \pm 1.5$ & $1.7 \pm 1.2$ & 0 & $2.3 \pm 0.6$ \\
\hline \multicolumn{6}{|l|}{ Cirrhitidae } \\
\hline Cirrhitus pinnulatus & 0 & $1.0 \pm 1.0$ & 0 & 0 & $1.7 \pm 0.6$ \\
\hline Paracirrhites forsteri & $3.7 \pm 1.2$ & $5.3 \pm 0.6$ & 0 & $4.3 \pm 1.2$ & $5.7 \pm 1.2$ \\
\hline \multicolumn{6}{|l|}{ Pseudochromidae } \\
\hline Pseudochromis flavivertex & 0 & $4.7 \pm 1.5$ & $0.3 \pm 0.6$ & 0 & $5.0 \pm 1.7$ \\
\hline Pseudochromis fridmani & 0 & $5.3 \pm 0.6$ & 0 & 0 & $5.3 \pm 0.6$ \\
\hline Pseudoplesiops auratus & 0 & $4.7 \pm 2.9$ & 0 & 0 & $5.3 \pm 0.6$ \\
\hline \multicolumn{6}{|l|}{ Carangidae } \\
\hline Carangoides bajad & $1.0 \pm 1.0$ & $12.3 \pm 1.5$ & $2.7 \pm 0.6$ & $1.3 \pm 0.6$ & $14.3 \pm 3.5$ \\
\hline Carangoides fulvogutatus & $2.0 \pm 1.0$ & $6.0 \pm 1.0$ & $0.7 \pm 1.2$ & $2.3 \pm 0.6$ & $7.3 \pm 1.5$ \\
\hline \multicolumn{6}{|l|}{ Lutjanidae } \\
\hline Lutjanus ehrenhergi & $0.7 \pm 1.2$ & $4.3 \pm 0.6$ & 0 & $1.3 \pm 1.2$ & $5.3 \pm 1.5$ \\
\hline Lutjanus fulviflamma & 0 & $3.0 \pm 1.0$ & $2.7 \pm 0.6$ & 0 & $3.7 \pm 2.1$ \\
\hline Lutjanus monostigma & $0.3 \pm 0.6$ & $2.7 \pm 0.6$ & $0.7 \pm 1.2$ & $1.7 \pm 2.9$ & $3.3 \pm 1.5$ \\
\hline Lutjanus kasmira & 0 & $12.7 \pm 2.1$ & 0 & 0 & $13.7 \pm 1.5$ \\
\hline Lutjanus bohar & 0 & $3.3 \pm 1.5$ & $1.3 \pm 1.2$ & 0 & $5.3 \pm 0.6$ \\
\hline \multicolumn{6}{|l|}{ Caesionidae } \\
\hline Caesio lunaris & $3.3 \pm 3.1$ & $18.7 \pm 2.5$ & $1.3 \pm 1.2$ & $4.3 \pm 2.1$ & $19.7 \pm 1.5$ \\
\hline Caesio suevicus & 0 & $24.7 \pm 3.8$ & $5.7 \pm 1.5$ & 0 & $26.7 \pm 3.2$ \\
\hline Caesio striatus & $1.3 \pm 2.3$ & $7.7 \pm 5.5$ & 0 & $1.3 \pm 1.5$ & $8.3 \pm 4.9$ \\
\hline Pterocaesio chrysozona & $1.0 \pm 1.7$ & $16.0 \pm 4.6$ & 0 & $1.3 \pm 1.2$ & $18.7 \pm 3.2$ \\
\hline \multicolumn{6}{|l|}{ Lethrinidae } \\
\hline Lethrinus harak & $1.7 \pm 1.5$ & $7.0 \pm 2.0$ & 0 & $1.3 \pm 1.2$ & $8.0 \pm 3.0$ \\
\hline Lethrinus lentjan & 0 & $3.7 \pm 2.1$ & 0 & 0 & $4.0 \pm 1.7$ \\
\hline Lethrinus mahsenoides & 0 & $0.7 \pm 1.2$ & $0.7 \pm 1.2$ & 0 & $1.3 \pm 1.2$ \\
\hline Lethrinus mahsena & 0 & $0.7 \pm 1.2$ & $1.7 \pm 0.6$ & 0 & $0.7 \pm 1.2$ \\
\hline Lethrinus nebulosus & 0 & $0.3 \pm 0.6$ & $1.3 \pm 0.6$ & 0 & $0.7 \pm 0.6$ \\
\hline Lethrinus lethrinus & 0 & $0.3 \pm 0.6$ & 0 & 0 & $0.7 \pm 1.2$ \\
\hline Monotaxis grandoculis & $0.3 \pm 0.6$ & $0.7 \pm 1.2$ & $0.7 \pm 1.2$ & $1.0 \pm 1.7$ & $1.3 \pm 0.6$ \\
\hline \multicolumn{6}{|l|}{ Sparidae } \\
\hline Rhabdosargus haffara & $0.3 \pm 0.6$ & $0.3 \pm 0.6$ & $17.0 \pm 4.6$ & $0.3 \pm 0.6$ & $0.3 \pm 0.6$ \\
\hline Rhabdosargus sarba & $0.3 \pm 0.6$ & $1.0 \pm 1.0$ & $3.7 \pm 1.5$ & $0.7 \pm 0.6$ & $1.3 \pm 1.5$ \\
\hline Acanthopagrus bifasciatus & 0 & $0.7 \pm 0.6$ & $2.3 \pm 2.1$ & 0 & $1.3 \pm 1.5$ \\
\hline Diplodus noct & $0.7 \pm 1.2$ & $3.0 \pm 1.7$ & $4.0 \pm 3.6$ & $0.7 \pm 1.2$ & $3.7 \pm 2.1$ \\
\hline \multicolumn{6}{|l|}{ Mullidae } \\
\hline Parupeneus macronema & $0.3 \pm 0.6$ & $0.3 \pm 0.6$ & $0.7 \pm 1.2$ & $0.3 \pm 0.6$ & $1.0 \pm 1.0$ \\
\hline Parupeneus forsskali & $3.7 \pm 3.2$ & $3.7 \pm 3.2$ & $35.3 \pm 11.9$ & $0.7 \pm 0.6$ & $3.7 \pm 3.2$ \\
\hline Parupeneus cyclostomus & 0 & $4.7 \pm 2.5$ & $1.3 \pm 2.3$ & 0 & $5.0 \pm 2.6$ \\
\hline
\end{tabular}


Table 1: continued.

\begin{tabular}{|c|c|c|c|c|c|}
\hline \multirow{2}{*}{ Fish species } & \multicolumn{2}{|c|}{ Sector A } & \multirow{2}{*}{$\frac{\text { Sector B }}{\text { Seagrass }}$} & \multicolumn{2}{|c|}{ Sector C } \\
\hline & $\mathrm{RF}$ & RW & & $\mathrm{RF}$ & RW \\
\hline Parupeneus rubescens & $0.7 \pm 1.2$ & $2.0 \pm 3.5$ & $0.7 \pm 1.2$ & $1.3 \pm 1.2$ & $2.0 \pm 3.5$ \\
\hline Mulloides flavolineatus & $1.7 \pm 2.9$ & $12.7 \pm 4.0$ & $17.3 \pm 6.5$ & $1.3 \pm 2.3$ & $12.7 \pm 5.5$ \\
\hline Mulloides vanicolensis & $1.0 \pm 1.7$ & $10.7 \pm 1.5$ & $22.7 \pm 7.5$ & $1.3 \pm 1.5$ & $14.0 \pm 2.6$ \\
\hline \multicolumn{6}{|l|}{ Mugilidae } \\
\hline Crenimugil crenilabis & 0 & $0.7 \pm 1.2$ & $3.3 \pm 0.6$ & $0.7 \pm 1.2$ & $1.0 \pm 1.7$ \\
\hline Oedalechilus labiosus & 0 & $1.7 \pm 2.9$ & $4.3 \pm 2.1$ & 0 & $1.7 \pm 2.1$ \\
\hline \multicolumn{6}{|l|}{ Pomacentridae } \\
\hline Amphiprion bicinctus & $0.7 \pm 1.2$ & $4.3 \pm 2.1$ & $1.3 \pm 1.2$ & $1.0 \pm 1.0$ & $4.7 \pm 1.5$ \\
\hline Dascyllus trimaculatus & $1.0 \pm 1.0$ & $4.7 \pm 0.6$ & 0 & $1.7 \pm 0.6$ & $5.3 \pm 1.5$ \\
\hline Dascyllus marginatus & $1.0 \pm 1.0$ & $5.0 \pm 1.0$ & $0.3 \pm 0.6$ & $1.3 \pm 1.5$ & $4.7 \pm 0.6$ \\
\hline Dascyllus aruanus & $2.3 \pm 1.5$ & $5.0 \pm 2.0$ & 0 & $2.7 \pm 1.2$ & $4.3 \pm 2.5$ \\
\hline Chromis dimidiata & $19.3 \pm 1.2$ & $166.7 \pm 12.6$ & $5.7 \pm 2.1$ & $16.7 \pm 2.9$ & $\begin{array}{c}178.3 \pm 25 \\
7\end{array}$ \\
\hline Chromis pelloura & 0 & $4.0 \pm 1.0$ & $1.7 \pm 1.2$ & 0 & $4.3 \pm 1.5$ \\
\hline Chromis pembae & 0 & $2.3 \pm 1.2$ & 0 & 0 & $2.3 \pm 1.2$ \\
\hline Chromis weberi & 0 & $3.0 \pm 1.0$ & $1.3 \pm 0.6$ & 0 & $3.0 \pm 1.0$ \\
\hline Chromis ternatensis & $0.7 \pm 0.6$ & $2.0 \pm 1.0$ & $0.3 \pm 0.6$ & $1.0 \pm 1.0$ & $2.0 \pm 1.0$ \\
\hline Chromis trialpha & 0 & $1.3 \pm 1.2$ & 0 & 0 & $1.3 \pm 1.2$ \\
\hline Chromis caerulea & 0 & $1.3 \pm 1.2$ & 0 & 0 & $1.3 \pm 1.2$ \\
\hline Pristotis cyanostigma & $1.7 \pm 1.5$ & 0 & 0 & $1.7 \pm 1.5$ & 0 \\
\hline Pomacentrus sulfureus & $3.0 \pm 1.0$ & $17.0 \pm 2.6$ & 0 & $3.7 \pm 2.1$ & $18.3 \pm 2.1$ \\
\hline Pomacentrus aquilus & $2.0 \pm 1.0$ & $2.3 \pm 1.5$ & 0 & $2.3 \pm 1.5$ & $2.3 \pm 1.5$ \\
\hline Pomacentrus albicaudtus & $1.7 \pm 0.6$ & $4.7 \pm 1.5$ & 0 & $1.7 \pm 0.6$ & $4.3 \pm 2.1$ \\
\hline Pomacentrus trilineatus & $1.7 \pm 0.6$ & $2.7 \pm 2.1$ & 0 & $2.3 \pm 1.5$ & $2.7 \pm 2.1$ \\
\hline Pomacentrus leptus & $0.7 \pm 0.6$ & $1.7 \pm 0.6$ & 0 & $0.7 \pm 0.6$ & $2.7 \pm 1.2$ \\
\hline Pomacentrus trichourus & $1.7 \pm 0.6$ & 0 & 0 & $2.0 \pm 1.0$ & $1.3 \pm 1.2$ \\
\hline Stegastes nigricans & $2.7 \pm 3.1$ & $7.0 \pm 4.0$ & 0 & $2.3 \pm 2.5$ & $8.0 \pm 2.6$ \\
\hline Plectroglyphidodon lacrymatus & 0 & 0 & 0 & 0 & 0 \\
\hline Plectroglyphidodon leucozona & $13.0 \pm 9.0$ & $4.7 \pm 1.5$ & 0 & $13.0 \pm 9.0$ & $5.3 \pm 0.6$ \\
\hline Paraglyphidodon melas & $2.3 \pm 4.0$ & $5.3 \pm 3.5$ & 0 & $2.3 \pm 4.0$ & $6.3 \pm 2.3$ \\
\hline Chrysiptera annulata & 0 & $5.3 \pm 2.5$ & 0 & 0 & $5.7 \pm 3.1$ \\
\hline Chrysiptera unimaculata & $3.7 \pm 3.2$ & $1.0 \pm 1.7$ & 0 & $4.0 \pm 2.6$ & $1.3 \pm 1.5$ \\
\hline Amblyglyphidon leucogaster & $0.7 \pm 1.2$ & $4.0 \pm 1.0$ & $2.7 \pm 0.6$ & $0.7 \pm 1.2$ & $4.3 \pm 0.6$ \\
\hline Amblyglyphidon flavilatus & $1.3 \pm 1.2$ & $5.0 \pm 1.0$ & 0 & $2.3 \pm 0.6$ & $5.7 \pm 0.6$ \\
\hline Abudefduf saxatilis & $6.0 \pm 1.0$ & $74.3 \pm 8.1$ & 0 & $6.3 \pm 1.5$ & $81.7 \pm 12.6$ \\
\hline Abudefduf sexfasciatus & 0 & $5.7 \pm 6.0$ & 0 & 0 & $6.7 \pm 4.7$ \\
\hline Abudefduf sordidus & 0 & $1.3 \pm 1.2$ & $2.7 \pm 0.6$ & 0 & $1.0 \pm 1.0$ \\
\hline \multicolumn{6}{|l|}{ Labridae } \\
\hline Bodianus anthioides & $0.7 \pm 0.6$ & 0 & $1.3 \pm 1.5$ & $1.0 \pm 1.0$ & 0 \\
\hline Cheilinus mentalis & 0 & $0.7 \pm 0.6$ & $1.3 \pm 0.6$ & 0 & $1.3 \pm 0.6$ \\
\hline Cheilinus undulatus & 0 & $0.3 \pm 0.6$ & 0 & 0 & $0.3 \pm 0.6$ \\
\hline Cheilinus lunulatus & 0 & $0.7 \pm 0.6$ & $0.3 \pm 0.6$ & 0 & $1.0 \pm 1.0$ \\
\hline Cheilinus fasciatus & 0 & 0 & 0 & 0 & $0.3 \pm 0.6$ \\
\hline Cheilinus abudjubbe & $0.3 \pm 0.6$ & $1.3 \pm 1.5$ & 0 & $0.3 \pm 0.6$ & $1.7 \pm 1.2$ \\
\hline Epibulus insidiator & $0.3 \pm 0.6$ & $2.3 \pm 0.6$ & 0 & $0.7 \pm 0.6$ & $2.7 \pm 0.6$ \\
\hline
\end{tabular}


Table 1: continued.

\begin{tabular}{|c|c|c|c|c|c|}
\hline \multirow{2}{*}{ Fish species } & \multicolumn{2}{|c|}{ Sector A } & \multirow{2}{*}{$\begin{array}{l}\text { Sector B } \\
\text { Seagrass }\end{array}$} & \multicolumn{2}{|c|}{ Sector C } \\
\hline & $\mathrm{RF}$ & RW & & RF & RW \\
\hline Novaculichthys taeniourus & 0 & $0.3 \pm 0.6$ & 0 & 0 & $0.3 \pm 0.6$ \\
\hline Pseudocheilinus hexataenia & 0 & $1.3 \pm 1.2$ & 0 & 0 & $1.3 \pm 1.2$ \\
\hline Labroides dimidiatus & $9.3 \pm 3.2$ & $15.0 \pm 2.6$ & 0 & $9.0 \pm 3.2$ & $18.0 \pm 2.6$ \\
\hline Larabicus quadrilineatus & 0 & $2.0 \pm 1.0$ & 0 & 0 & $2.0 \pm 1.0$ \\
\hline Coris gaimard & 0 & 0 & $2.3 \pm 0.6$ & 0 & $0.7 \pm 1.2$ \\
\hline Coris aygula & 0 & $0.7 \pm 0.6$ & $0.7 \pm 0.6$ & 0 & $1.0 \pm 1.0$ \\
\hline Hologymnosus annulatus & 0 & $0.3 \pm 0.6$ & $0.3 \pm 0.6$ & 0 & $0.3 \pm 0.6$ \\
\hline Hemigymnus fasciatus & 0 & $1.0 \pm 1.0$ & 0 & 0 & $1.0 \pm 1.0$ \\
\hline Thalassoma rueppellii & $55.3 \pm 23.1$ & $59.7 \pm 26.3$ & $1.3 \pm 1.2$ & $58.3 \pm 17.6$ & $65.0 \pm 30.4$ \\
\hline Gomphosus coeruleus & $1.3 \pm 1.5$ & $3.3 \pm 0.6$ & $0.3 \pm 0.6$ & $1.3 \pm 1.5$ & $4.0 \pm 1.7$ \\
\hline Pseudodax moluccanus & 0 & $1.3 \pm 0.6$ & 0 & 0 & $1.7 \pm 0.6$ \\
\hline \multicolumn{6}{|l|}{ Sphyraenidae } \\
\hline Sphyraena barracuda & 0 & $5.3 \pm 3.1$ & 0 & 0 & $6.3 \pm 3.5$ \\
\hline Sphyraena jello & 0 & $1.3 \pm 1.2$ & 0 & 0 & $1.7 \pm 0.6$ \\
\hline Sphyraena putnamiae & 0 & $1.7 \pm 1.5$ & 0 & 0 & $2.3 \pm 0.6$ \\
\hline \multicolumn{6}{|l|}{ Scaridae } \\
\hline Hipposcarus harid & $1.3 \pm 1.2$ & $3.3 \pm 0.6$ & $0.3 \pm 0.6$ & $1.7 \pm 0.6$ & $4.0 \pm 1.0$ \\
\hline Cetoscarus bicolor & 0 & $0.3 \pm 0.6$ & 0 & 0 & $0.3 \pm 0.6$ \\
\hline Scarus sordidus & $8.3 \pm 0.6$ & $2.7 \pm 0.6$ & 0 & $6.3 \pm 1.5$ & $2.7 \pm 0.6$ \\
\hline Scarus gibbus & 0 & $0.3 \pm 0.6$ & 0 & 0 & $0.3 \pm 0.6$ \\
\hline Scarus ghobban & $2.7 \pm 0.6$ & $0.7 \pm 1.2$ & 0 & $3.3 \pm 1.5$ & $0.7 \pm 1.2$ \\
\hline Scarus ferrugineus & 0 & $0.7 \pm 0.6$ & $0.3 \pm 0.6$ & 0 & $0.7 \pm 0.6$ \\
\hline Scarus psittacus & 0 & 0 & $2.3 \pm 0.6$ & 0 & 0 \\
\hline Scarus frenatus & $0.7 \pm 0.6$ & $1.7 \pm 0.6$ & 0 & $1.3 \pm 0.6$ & $2.3 \pm 0.6$ \\
\hline Scarus collana & 0 & $0.7 \pm 0.6$ & 0 & 0 & $0.7 \pm 0.6$ \\
\hline Scarus niger & $1.0 \pm 1.0$ & $0.7 \pm 1.2$ & $0.7 \pm 0.6$ & $1.0 \pm 1.0$ & $1.3 \pm 1.5$ \\
\hline \multicolumn{6}{|l|}{ Chaetodontidae } \\
\hline Chaetodon auriga & $2.3 \pm 0.6$ & $5.0 \pm 1.0$ & $2.7 \pm 2.5$ & $3.0 \pm 1.0$ & $5.0 \pm 1.0$ \\
\hline Chaetodon fasciatus & $3.3 \pm 1.2$ & $6.0 \pm 1.0$ & $1.7 \pm 1.2$ & $3.7 \pm 2.5$ & $4.7 \pm 1.5$ \\
\hline Chaetodon austriacus & $5.7 \pm 0.6$ & $11.3 \pm 1.5$ & $0.7 \pm 1.2$ & $4.3 \pm 1.5$ & $12.0 \pm 3.0$ \\
\hline Chaetodon melannotus & 0 & $1.7 \pm 0.6$ & 0 & 0 & $1.7 \pm 0.6$ \\
\hline Chaetodon paucifasciatus & 0 & $1.0 \pm 1.0$ & $0.3 \pm 0.6$ & 0 & $1.0 \pm 1.0$ \\
\hline Megaprotodon trifascialis & $2.3 \pm 0.6$ & $7.7 \pm 1.5$ & 0 & $2.7 \pm 1.2$ & $7.7 \pm 2.5$ \\
\hline Gonochaetodon larvatus & $0.3 \pm 0.6$ & 0 & $0.7 \pm 1.2$ & $0.3 \pm 0.6$ & 0 \\
\hline Heniochus intermedius & $3.0 \pm 1.0$ & $7.7 \pm 1.5$ & $1.3 \pm 1.2$ & $2.7 \pm 1.5$ & $6.3 \pm 1.5$ \\
\hline Heniochus diphreutes & 0 & 0 & $0.7 \pm 1.2$ & 0 & 0 \\
\hline \multicolumn{6}{|l|}{ Pomacanthidae } \\
\hline Centropyge multispinis & $3.0 \pm 1.0$ & $5.7 \pm 1.2$ & $1.3 \pm 0.6$ & $3.3 \pm 0.6$ & $5.7 \pm 1.2$ \\
\hline Pygoplites diacanthus & $2.0 \pm 1.0$ & $7.7 \pm 3.8$ & $0.7 \pm 1.2$ & $2.7 \pm 1.5$ & $8.7 \pm 1.5$ \\
\hline Genicanthus cadovittatus & 0 & $2.0 \pm 1.0$ & 0 & 0 & $2.3 \pm 2.1$ \\
\hline \multicolumn{6}{|l|}{ Acanthuridae } \\
\hline Zebrasoma desjardinii & $26.7 \pm 9.3$ & $10.7 \pm 3.2$ & $2.3 \pm 0.6$ & $26.7 \pm 7.6$ & $10.0 \pm 5.0$ \\
\hline Zebrasoma xanthurum & $3.3 \pm 1.5$ & $7.7 \pm 0.6$ & $0.3 \pm 0.6$ & $3.7 \pm 1.2$ & $5.0 \pm 3.0$ \\
\hline Acanthurus nigricans & 0 & $3.7 \pm 2.1$ & 0 & 0 & $4.0 \pm 2.0$ \\
\hline
\end{tabular}


Table 1: continued.

\begin{tabular}{|c|c|c|c|c|c|}
\hline \multirow{2}{*}{ Fish species } & \multicolumn{2}{|c|}{ Sector A } & \multirow{2}{*}{$\begin{array}{l}\text { Sector B } \\
\text { Seagrass }\end{array}$} & \multicolumn{2}{|c|}{ Sector C } \\
\hline & $\mathrm{RF}$ & RW & & $\mathrm{RF}$ & RW \\
\hline Acanthurus nigrofuscus & $27.0 \pm 7.9$ & $6.3 \pm 3.1$ & $0.7 \pm 1.2$ & $27.0 \pm 7.9$ & $5.7 \pm 3.1$ \\
\hline Acanthurus sohal & $37.7 \pm 3.8$ & $8.7 \pm 2.1$ & 0 & $35.0 \pm 5.0$ & $6.7 \pm 1.5$ \\
\hline Ctenochaetus striatus & 0 & 0 & $2.3 \pm 0.6$ & 0 & 0 \\
\hline Naso lituratus & $0.3 \pm 0.6$ & $5.0 \pm 1.0$ & 0 & $1.0 \pm 1.0$ & $5.7 \pm 0.6$ \\
\hline Naso unicornis & 0 & $16.0 \pm 9.6$ & $1.7 \pm 1.5$ & 0 & $18.3 \pm 7.8$ \\
\hline \multicolumn{6}{|l|}{ Siganidae } \\
\hline Siganus rivulatus & $1.0 \pm 1.7$ & 0 & $13.3 \pm 5.1$ & $2.3 \pm 1.2$ & $1.0 \pm 1.7$ \\
\hline Siganus argenteus & $0.7 \pm 1.2$ & 0 & $1.7 \pm 0.6$ & $1.7 \pm 0.6$ & 0 \\
\hline \multicolumn{6}{|l|}{ Balistidae } \\
\hline Balistapus undulatus & 0 & $1.3 \pm 0.6$ & 0 & 0 & $2.7 \pm 2.1$ \\
\hline Sufflamen albicaudatus & 0 & $0.3 \pm 0.6$ & $2.3 \pm 0.6$ & 0 & $0.3 \pm 0.6$ \\
\hline Rhinecanthus assasi & $1.3 \pm 0.6$ & $1.3 \pm 1.5$ & $2.7 \pm 1.2$ & $2.3 \pm 0.6$ & $1.7 \pm 1.2$ \\
\hline Pseudobalistes fuscus & 0 & $0.7 \pm 0.6$ & $0.7 \pm 1.2$ & 0 & $1.3 \pm 0.6$ \\
\hline \multicolumn{6}{|l|}{ Ostraciidae } \\
\hline Ostracion cyanurus & 0 & $1.3 \pm 0.6$ & $2.3 \pm 0.6$ & 0 & $1.7 \pm 0.6$ \\
\hline Tetrosomus gibbosus & 0 & $0.3 \pm 0.6$ & $0.7 \pm 1.2$ & 0 & $1.3 \pm 1.5$ \\
\hline \multicolumn{6}{|l|}{ Haemulidae } \\
\hline Plectorhynchus gaterinus & 0 & $0.7 \pm 0.6$ & $2.3 \pm 0.6$ & 0 & $2.3 \pm 1.5$ \\
\hline Plectorhynchus pictus & 0 & $0.7 \pm 1.2$ & $1.7 \pm 1.2$ & 0 & $1.7 \pm 0.6$ \\
\hline \multicolumn{6}{|l|}{ Scorpaenidae } \\
\hline Synanceia verrucosa & $0.7 \pm 0.6$ & 0 & 0 & $1.0 \pm 1.0$ & 0 \\
\hline Pterois volitans & 0 & $0.3 \pm 0.6$ & $0.7 \pm 1.2$ & 0 & $0.7 \pm 1.2$ \\
\hline Pterois radiata & $0.3 \pm 0.6$ & $2.0 \pm 1.0$ & $0.3 \pm 0.6$ & $0.7 \pm 1.2$ & $2.0 \pm 1.0$ \\
\hline \multicolumn{6}{|l|}{ Tetraodontidae } \\
\hline Canthigaster coronata & 0 & $1.0 \pm 1.0$ & 0 & 0 & $1.3 \pm 0.6$ \\
\hline Arothron diadematus & $0.3 \pm 0.6$ & $1.0 \pm 1.0$ & $5.3 \pm 2.1$ & $0.3 \pm 0.6$ & $1.3 \pm 1.5$ \\
\hline Arothron hispidus & 0 & $0.7 \pm 0.6$ & $1.3 \pm 1.2$ & 0 & $1.0 \pm 1.0$ \\
\hline \multicolumn{6}{|l|}{ Apogonidae } \\
\hline Apogon exostigma & 0 & $0.7 \pm 1.2$ & 0 & 0 & $1.0 \pm 1.0$ \\
\hline Apogon cookii & $0.3 \pm 0.6$ & 0 & $0.3 \pm 0.6$ & $0.3 \pm 0.6$ & $0.7 \pm 1.2$ \\
\hline Apogon cyanosoma & $0.7 \pm 0.6$ & $1.7 \pm 0.6$ & $0.3 \pm 0.6$ & $0.7 \pm 0.6$ & $2.3 \pm 0.6$ \\
\hline \multicolumn{6}{|l|}{ Gobiidae } \\
\hline Amblyeleotris steinitzi & 0 & $3.0 \pm 1.7$ & $0.3 \pm 0.6$ & $0.7 \pm 1.2$ & $2.7 \pm 0.6$ \\
\hline Amblyeleotris sungami & 0 & $3.3 \pm 0.6$ & $0.7 \pm 1.2$ & $1.7 \pm 1.2$ & $4.3 \pm 1.5$ \\
\hline Istigobius decoratus & 0 & $2.0 \pm 1.7$ & 0 & $1.3 \pm 1.5$ & $2.0 \pm 1.7$ \\
\hline Amblygobius albimaculatus & 0 & 0 & $1.3 \pm 1.2$ & 0 & $1.0 \pm 1.0$ \\
\hline Diodontidae & & & & & \\
\hline Chilomycterus spilostylus & 0 & 0 & $0.3 \pm 0.6$ & 0 & $0.3 \pm 0.6$ \\
\hline
\end{tabular}

The highest average abundance was recorded at sector $C$ on reef wall habitat (1119 fish/1000 $\mathrm{m}^{3}$, Table 2), with the lowest value at sector B on seagrass habitat $\left(240 \mathrm{fish} / 1000 \mathrm{~m}^{3}\right)$. Some species were restricted to seagrass or any zone in coral reefs (i.e., shallow back reef, shallow fore reef, inner reef and outer reef), while others were widespread along the gradient. In terms of relative abundance per habitats, there were some species that dominated over others. Species were restricted to coral reefs: Adioryx diadema, Anthias squamipinnis, 
Carangoides bajad, Pterocaesio chrysozoma, Pomacentrus sulfreus and Thalassoma rueppellii. Others were restricted to seagrasses: Parupeneus forsskali, Mulloides flavolineatus, M. vanicolensis, Rhabdosargus haffara and Siganus rivulatus.

Table 2: Diversity indices and characteristics at each sector in Marsa Abu Dabab, Red Sea.

\begin{tabular}{|l|ccccc|}
\hline & \multicolumn{2}{|c}{ Sector A } & Sector B & \multicolumn{2}{c|}{ Sector C } \\
\cline { 2 - 6 } & RF & RW & Seagrass & RF & RW \\
\hline Number of species & 81 & 153 & 90 & 88 & 162 \\
Number of individuals & 319 & 1062 & 240 & 346 & 1119 \\
Species richness (D) & 13.88 & 21.81 & 16.25 & 14.88 & 22.93 \\
Evenness $\left(\mathrm{J}^{\prime}\right)$ & 0.753 & 0.743 & 0.819 & 0.791 & 0.765 \\
Shannon-Wiener $\left(\mathrm{H}^{\prime}\right)$ & 3.31 & 3.74 & 3.69 & 3.54 & 3.89 \\
\hline
\end{tabular}

The average species richness ranged from 13.88 at sector A on reef flat to 22.93 at sector $C$ on reef wall (Table 2). The highest evenness index $\left(\mathrm{J}^{\prime}\right)$ was recorded at sector B in seagrass beds (0.819), while sector A on reef wall yielded the lowest value $(0.743)$. The average Shannon-Wiener diversity $\left(\mathrm{H}^{\prime}\right)$ varied between 3.31 at sector $\mathrm{A}$ on reef flat and 3.89 at sector $\mathrm{C}$ on reef wall. The mean diversity and mean species richness along the gradient were significantly different among the investigated habitats $(p=0.001)$.

\section{Distribution patterns of fish families}

Figure (3) shows distribution patterns of eight fish families (Pomacentridae, Labridae, Acanthuridae, Chaetodontidae, Serranidae, Siganidae, Sparidae and Mullidae) over different habitats at 5 study sites in Marsa Abu Dabab. Five families showed increasing in coral reef sites (Pomacentridae, Labridae, Acanthuridae, Chaetodontidae and Serranidae), while other three families increased in seagrass beds (Siganidae, Sparidae and Mullidae; Figure $3)$. The recorded five fish families in coral reef sites showed high abundance on reef wall (RW) more than on reef flat (RF), except Acanthuridae. The fish assemblage at the coral reef habitats (sector A and C) was dominated by Acanthuridae, Pomacentridae, Labridae Syngnathidae and Scaridae (Table 3) on reef flat. While on reef wall, Pomacentridae, Serranidae and Labridae were dominated. Mullidae, Sparidae, Pomacentridae and Siganidae were dominant mostly in seagrass beds (sector B). 

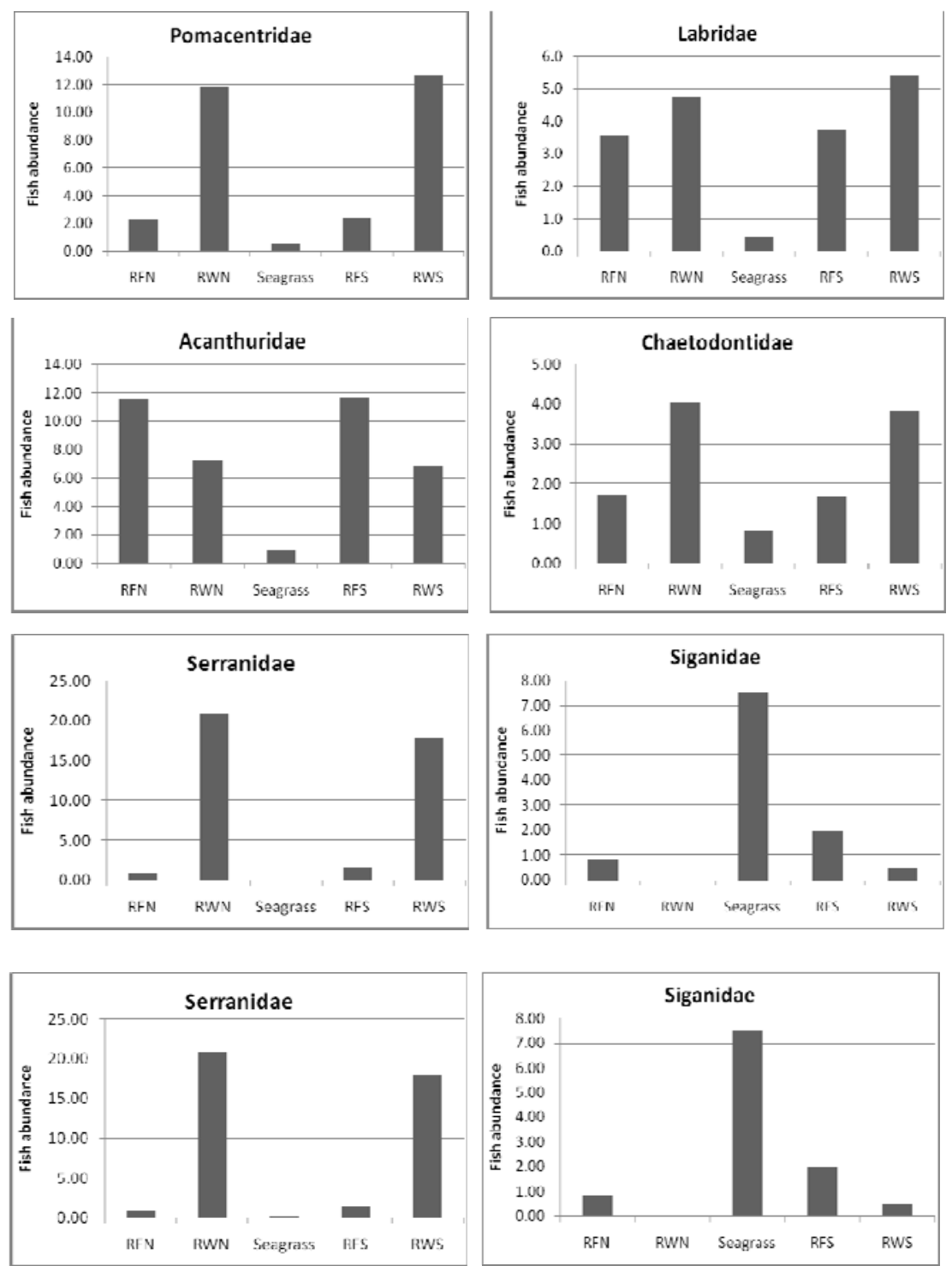

Fig. 3: Distribution patterns of eight common fish families (Pomacentridae, Labridae, Acanthuridae, Chaetodontidae, Serranidae, Siganidae, Sparidae and Mullidae) at Marsa Abu Dabab in each sector (Sector A including reef flat: REN, reef wall: RWN; Sectors B including seagrass beds; Sector C including reef flat: RFS, reef wall: RWS). 
Table 3: Percentage of occurrence of fish families at Marsa Abu Dabab in each sector.

\begin{tabular}{|c|c|c|c|c|c|c|}
\hline \multirow{2}{*}{ Fish family } & \multirow{2}{*}{ Common name } & \multicolumn{2}{|c|}{ Sector A } & \multirow{2}{*}{$\begin{array}{c}\text { Sector B } \\
\text { Seagr. }\end{array}$} & \multicolumn{2}{|c|}{ Sector C } \\
\hline & & $\mathrm{RF}$ & RW & & $\mathrm{RF}$ & RW \\
\hline Dasyatididae & Stingrays & 0 & 0.35 & 0.56 & 0 & 0.27 \\
\hline Synodontidae & Lizardfishes & 0.31 & 0.97 & 0.69 & 0.58 & 1.01 \\
\hline Muraenidae & Morays & 0.10 & 0.16 & 0 & 0.29 & 0.21 \\
\hline Belonidae & Needlefishes & 0 & 0.53 & 0 & 0.19 & 0.51 \\
\hline Hemiramphidae & Halfbeaks & 0 & 0.50 & 3.19 & 0 & 0.42 \\
\hline Atherinidae & Silversides & 0 & 0 & 1.53 & 0 & 0.12 \\
\hline Fistulariidae & Cornetfishes & 0 & 0.22 & 0.28 & 0 & 0.21 \\
\hline Syngnathidae & Pipefishes & 5.95 & 0.16 & 1.94 & 3.47 & 0.80 \\
\hline Antennariidae & Frogfishes & 0.21 & 0.03 & 0.56 & 0.10 & 0.03 \\
\hline Anomalopidae & Flashlight fishes & 0 & 0.16 & 0 & 0 & 0.06 \\
\hline Holocentridae & Squirrelfishes & 0 & 2.29 & 0 & 0.58 & 2.14 \\
\hline Serranidae & Groupers & 2.93 & 21.56 & 0.42 & 5.01 & 17.69 \\
\hline Priacanthidae & Bigeyes & 0 & 0.16 & 0.69 & 0 & 0.21 \\
\hline Cirrhitidae & Hawkfishes & 1.15 & 0.59 & 0 & 1.25 & 0.66 \\
\hline Pseudochromidae & Dottybacks & 0 & 1.38 & 0.14 & 0 & 1.40 \\
\hline Carangidae & Jacks & 0.94 & 1.72 & 1.39 & 1.06 & 1.94 \\
\hline Lutjanidae & Snappers & 0.31 & 2.45 & 1.94 & 0.87 & 2.80 \\
\hline Caesionidae & Fusiliers & 1.77 & 6.31 & 2.92 & 2.02 & 6.55 \\
\hline Lethrinidae & Emperors & 0.62 & 1.26 & 1.81 & 0.67 & 1.49 \\
\hline Sparidae & Porgies & 0.41 & 0.47 & 11.25 & 0.48 & 0.60 \\
\hline Mullidae & Goatfishes & 1.24 & 3.20 & 32.50 & 1.45 & 3.43 \\
\hline Mugilidae & Mullets & 0 & 0.22 & 3.19 & 0.19 & 0.24 \\
\hline Pomacentridae & Damselfishes & 21.00 & 32.17 & 6.67 & 20.13 & 33.01 \\
\hline Labridae & Wrasses & 21.11 & 8.51 & 3.33 & 20.52 & 9.17 \\
\hline Sphyraenidae & Barracudas & 0 & 0.78 & 0 & 0 & 0.92 \\
\hline Scaridae & Parrotfishes & 4.41 & 1.04 & 1.53 & 3.95 & 1.16 \\
\hline Chaetodontidae & Butterflyfishes & 5.33 & 3.80 & 3.33 & 4.83 & 3.43 \\
\hline Pomacanthidae & Angelfishes & 1.57 & 1.44 & 0.83 & 1.73 & 1.49 \\
\hline Acanthuridae & Surgeonfishes & 28.84 & 5.46 & 3.06 & 26.97 & 4.94 \\
\hline Siganidae & Rabbitfishes & 0.52 & 0 & 6.25 & 1.16 & 0.09 \\
\hline Balistidae & Triggerfishes & 0.42 & 0.35 & 2.36 & 0.67 & 0.54 \\
\hline Ostraciidae & Trunkfishes & 0 & 0.16 & 1.25 & 0 & 0.27 \\
\hline Haemulidae & Grunts & 0 & 0.13 & 1.67 & 0 & 0.36 \\
\hline Scorpaenidae & Scorpionfishes & 0.31 & 0.33 & 0.42 & 0.48 & 0.24 \\
\hline Tetraodontidae & Puffers & 0.10 & 0.25 & 2.78 & 0.10 & 0.33 \\
\hline Apogonidae & Cardinalfishes & 0.31 & 0.22 & 0.28 & 0.29 & 0.36 \\
\hline Gobiidae & Gobies & 0 & 0.78 & 0.97 & 1.06 & 0.89 \\
\hline Diodontidae & Burrfishes & 0 & 0 & 0.14 & 0 & 0.03 \\
\hline
\end{tabular}

\section{DISCUSSION}

Fish assemblages associated with shallow, coastal areas with seagrass and coral reefs vary greatly at several spatial scales (Nagelkerken et al., 2000; 
Christensen et al., 2003; Faunce and Serafy, 2006). Coral reef fishes inhabit an environment characterized by great spatial heterogeneity in terms of substrate composition and structural complexity (Done, 1982; Rajasuriya et al., 1998). Fish may be selective or non-selective, obligate, facultative or opportunistic in relation to their habitat (Bergman et al., 2000).

Coral reef and seagrass habitats are subject to a wide range of anthropogenic impacts (Wilkinson, 1999; Leujak and Ormond, 2008). Direct anthropogenic impacts play a major role in devastating coral reefs (Hughes et al., 2003). One example is coastal tourism; tourism is now the world's largest single economic sector (Davenport and Davenport, 2006), indicating the threat it poses on coastal ecosystems world-wide. In Egypt, the number of tourists has steadily increased to 8.6 million in 2005 (OECD, 2006). Egypt's tourism sector is still expanding; the main investment target is the Red Sea region, in particular the South Sinai and Marsa Alam. Consequently, tourism centers like Sharm-elSheikh and Marsa Alam (including Abu Dabab) will continue to expand and new resorts will be built northwards along the coastline of the Egyptian Red Sea reefs. The result will be putting coral reef ecosystems in this area under increasing anthropogenic pressure. The role of human activity in shaping marine ecosystems is receiving increased attention with the realization that human activities are causing dramatic shifts in species composition and causing severe economic loss for local communities (Bellwood et al., 2004).

There were significant differences in the community structure and spatial distribution of fishes among coral reefs and seagrass beds. The fish community around Marsa Abu Dabab, i.e., coral reefs and seagrass beds, was typical of that found in other inshore areas of the northern Red Sea. In the present results, some species were restricted to seagrass or coral reefs, while others were widespread along the gradient. In terms of relative abundance per habitats, there were some species that dominated over others.

The mean fish abundance, mean species richness, and diversity along the cross-shelf gradient were higher at the coral reef habitats relative to the seagrass habitat (Aguilar-Perera and Appeldoorn, 2008). The total density for these strata was twice as high as that of the mangrove stratum. Plausible reasons explaining highest fish abundance in coral reefs compared to seagrass are that coral reefs offer greater structural complexity and availability of shelter (Roberts and Ormond, 1987; Friedlander and Parrish, 1998). Larval and juvenile recruitment in reef fish communities have an important role in determining the structure and stability of these communities. Settlement is influenced by habitat selection for substrate types (Williams and Sale, 1981), and many reef fish species prefer to settle on live corals (Booth and Beretta, 2002). Alwany et al. (2007) reported that the Pomacentridae was the highest abundant group of fishes in the coral reef in Sharm El-Maiya Bay in northern Red Sea. The present results confirm the previous finding, where the Pomacentridae represented by 21-33\% of the total fish population, belonging to 29 species. In addition, Jones (1997) 
found that juvenile growth and survival may be substantially affected by the structure of the habitat. Gab-Alla (2001) reported that the seagrass meadow in Sharm El-Maiya Bay has three species of seagrasses (Halodule uninervis, $H$. ovalis and Halophila stipulacea). In the present study, seagrass meadow Marsa Abu Dabab has four species of seagrasses (H. stipulacea, $H$. ovalis, $H$. uninervis and Syrngodium isotfolium). These four species occurred in moderately covers on the reef flat, while $H$. stipulacea was recorded in high covers inside the bay and at the sandy bottom near the reefs until 30-50 m depth.

Jennings et al. (1996) reported that the differences in the diversity and abundance of reef fish communities may also be attributed to spatial and temporal variations in recruitment (Doherty, 1991), habitat effects (Williams, 1991) and other factors. Habitat availability (Caley et al., 1996) and habitat preferences (Caley, 1995; Tolimieri, 1998) also play a role. One of the factors thought to be related to coral reef fish diversity is the heterogeneity of the organic and inorganic substrate that might be used by fish. Coral diversity and substrate heterogeneity can affect reef fish diversity by offering different opportunities for shelter, which directly influences larval recruitment and rates and patterns of colonization. Some researchers have found variation in species compositions with depth, or more generally among differing habitats (McGehee, 1994; Dominici-Arosemena et al., 2005). Various studies have indicated that physical variables, in particular substrate, depth, and currents, have an important influence on the distribution of fish and other aquatic organisms (Williams, 1982; Russ, 1984; McGehee, 1994).

\section{ACKNOWLEDGEMENTS}

The author thanks Dr. Maher A. Amar and Dr. Mohsen M. El-Sherbiny for their suggestions and encouragement in planning this study. This work has been done with the assistance of the Department of Marine Science, Suez Canal University, Ismailia, Egypt.

\section{REFERENCES}

Aguilar-Perera, A. and Appeldoorn, R. S. (2008). Spatial distribution of marine fishes along a cross-shelf gradient containing a continuum of mangroveseagrass-coral reefs off southwestern Puerto Rico. Estua. Coast. She. Sci., 76: 378-394.

Alwany, M. A.; Hanafy, M.; Kotb, M. and GabAlla A. A. (2007). Species diversity and habitat distribution of fishes in Sharm El-Maiya Bay, Sharm El-Sheikh, Red Sea. Catrina, 2(1): 83-90.

Bellwood, D. R.; Hughes, T. P.; Folke, C. and Nystrom, M. (2004). Confronting the coral reef crisis. Nature, 429: 827-833. 
Bergman, K. C.; Öhman, M. C. and Svensson, S. (2000). Influence of habitat structure on Pomacentrus sulfurous, a western Indian Ocean reef fish. Envi. Biol. Fish., 59: 243-252.

Birkeland, C. (1997). Life and death of coral reefs. Chapman and Hall, New York. pp.536.

Birkeland, C. (2004). Ratcheting down the coral reefs. Bioscience, 54:10211027.

Booth, D. J. and Beretta, G. A. (2002). Changes in a fish assemblage after a coral bleaching event. Mar. Ecol. Prog. Ser., 245: 205-212.

Caley, M. J. (1995). Reef-fish community structure and dynamics: an interaction between local and larger scale? Mar. Ecol. Prog. Ser. 129: 19-29.

Caley, M. J.; Carr, M.; Hiscor, H. M. A.; Huges, T. P.; Jones, G. P. and Menge, B. A. (1996). Recruitment and the local dynamics of open marine populations. Ann. Rev. Ecol. Syst., 27: 477-500.

Christensen, J. D.; Jeffrey, C. F. G.; Caldow, C.; Monaco, M. E.; Kendall, M. S. and Appeldoorn, R.S. (2003). Cross-shelf habitat utilization patterns of reef fishes in southwestern Puerto Rico. Gulf Caribb. Res., 14: 9-28.

Davenport, J. and Davenport, J. L. (2006). The impact of tourism and personal leisure transport on coastal environments: a review. Estua. Coast. She. Sci., 67: 280-292.

Doherty, P. J. (1991). Spatial and temporal patterns in recruitment. In: Sale, P.F. (ed), The ecology of fishes on coral reefs. Academic Press, San Diego: 261-293.

Dominici-Arosemena A.; Brugnoli-Olivera, E.; Solano-Ulate S., and RamírezCoghi., A. (2000). Ictioplancton en la zona portuaria de Limón, Costa Rica. Rev. Biol. Trop., 48(2):439-442.

Done T. J. (1982). Patterns in the distribution of coral communities across the Great Barrier Reef. Coral Reefs, 1: 95-107.

English, S.; Wilkinson, C. and Baker, V. (1997). Survey manual for tropical marine science, (second Ed.), Australian Institute of Marine Science, Townsville. 
Faunce, C. H. and Serafy, J. E. (2006). Mangroves as fish habitat: 50 years of field studies. Mar. Ecol. Prog. Ser., 318: 1-18.

Friedlander, A. M. and Parrish, J. D. (1998). Habitat characteristics affecting fish assemblages on a Hawaiian coral reef. J. Exp. Mar. Biol. Ecol., 224: $1-30$.

Gab-Alla, A. A. F. A. (2001). Ecological status of the seagrass community in Sharm El-Maiya Bay (Gulf of Aqaba, Red Sea) after oil pollution in 1999. J. King Abdulaziz University: Mar. Sci., 12 (2): 231-239.

Gillanders, B. M. (2006). Seagrasses, fish and fisheries. In: A.W. Larkum (eds.), Biology, ecology and Conservation, Springer, Netherlands, 503-536 pp.

Hughes T. P.; Baird, A. H. and Dinsdale, E. A. (2002). Detecting regional variation using meta-analysis and large scale sampling: latitudinal patterns in recruitment. Ecol., 83(2): 436-451.

Hughes, T. P.; Baird, A. H.; Bellwood, D. R.; Card, M.; Connolly, S. R.; Folke, C.; Grosberg, R.; Hoegh-Guldberg, O.; Jackson, J. B. C.; Kleypas, J.; Lough, J. M.; Marshall, P.; Nystrom, M.; Palumbi, S. R.; Pandolfi, J. M.; Rosen, B. and Roughgarden, J. (2003). Climate change, human impacts, and the resilience of coral reefs. Sci., 301: 929-933.

Jennings, S.; Boullé, D. and Polunin, N. V. C. (1996). Habitat correlates of the distribution and biomass of Seychelles reef fishes. Envi. Biol. Fish, 46: 15-25.

Jones, G. P. (1997). Relationships between recruitment and post-recruitment processes in lagoonal populations of two coral reef fishes. J. Exp. Mar. Biol. Ecol., 213: 231-246.

Karlson R H. and Cornell H.V., (1998). Scale-dependent variation in local vs. regional effects on coral species richness. Ecological Monographs, 68(2): 259-274.

Kotb, M. M. A.; Abdulaziz, M.; Al-Agwan, Z.; Alshaikh, K.; Al-Yami, H.; Banajah, A.; Devamed, L.; Eisinger, M.; Eltayeb, M.; Hassan, M.; Heiss, G.; Howe, S.; Kemp, J.; Klaus, R.; Krupp, F.; Mohamed, N.; Rouphael, T.; Turner, J. and Zaajonz, U. (2004). Status of coral reefs in the Red Sea and Gulf of Aden in 2004. In: Wilkinson, C. (Ed.), Status of coral reefs of the World. Australian Institute of Marine Science, Townsville, pp. 137-154. 
Leujak, W. and Ormond, R. F. G. (2008). Reef walking on Red Sea reef flats Quantifying impacts and identifying motives. Ocean Coast. Manag. 22 : $1-8$.

McGehee, A. (1994). Correspondence between assemblages of coral reef fishes and gradients of water motion depth, and substrate size off Puerto Rico. Mar. Ecol. Prog. Ser. 105:243-255.

Mora, C.; Chittaro, P. M.; Sale, P. F.; Kritzer, J. P. and Ludsin, S. A. (2003). Patterns and processes in reef fish diversity. Nature, 421: 933-936.

Nagelkerken, I.; van der Velde, G.; Gorissen; M. W.; Meijer, G. J.; van’t Hof, T. and den Hartog, C. (2000). Importance of mangroves, seagrass beds and the shallow coral reef as a nursery for important coral reef fishes, using a visual census technique. Estuarine, Coast. Shel. Sci., 51: 31-44.

OECD (2006). African Economic Outlook 2005-2006, Egypt, 252 pp.

Ormond, R. F. G. and Edwards, A. (1987). Red Sea fishes. In: Edwards, A.J., Head, S.M. (Eds.), Red Sea. Pergamon Press, Oxford, pp. 251-287.

Pandolifi, J. M.; Bradbury, R. H. and Sala, E. (2003). Global trajectories of the long-term decline of coral reef ecosystems. Sci., 301: 955-958.

Pattengill, C. V.; Semmens, B. X. and Gittings, S. R. (1997). Reef fish structure at the Flower Gardens and Stetson Bank, NW Gulf of Mexico. Proceeding of the $8^{\text {th }}$ International Coral Reef Symposium, 1:1023-1028.

Pielou, E. C. (1969). Shannon's formula as a measure of specific diversity. Its use and misuse. Amer. Natur., 100: 463-465.

Plotkin J. B. and Muller-Landau, H. C. (2002). Sampling the species composition of a landscape. Ecol., 83(12): 3344-3356.

Rajasuriya, A.; Öhman, M. C. and Johnstone, R. W. (1998). Coral and sandstone reef-habitats in southern Sri Lanka: patterns in the distribution of coral communities. Ambio, 27: 726-728.

Randall, J. E. (1983). In: Red Sea Reef Fishes. Immel Publishing, London, pp. 192. 
Roberts C. M. and Ormond, R. F. (1987). Habitat complexity and coral reef fish diversity and abundance on Red Sea fringing reefs. Mar. Ecol. Prog. Ser., 41: 1-8.

Roberts, C. M.; McClean, C. J.; Veron, J. E. N.; Hawkins, J. P.; Allen, G. R., McAllister, D. E.; Mittermeier, C. G.; Schueler, F. W.; Spalding, M.; Wells, F.; Vynne, C. and Werner, T. B. (2002). Marine biodiversity hotspots and conservation priorities for tropical reefs. Sci., 295: 12801284.

Russ, G. R. (1984). Distribution and abundance of herbivorous grazing fishes in the central Great Barrier Reef. II. Patterns of zonation of mid-shelf and outershelf. Mar. Ecol. Prog. Ser., 20: 35-44.

Sale, P. F. (1980). The ecology of fishes on coral reefs. Oceanography Marine Biology: Ann. Rev., 18: 367-421.

Tolimieri, N. (1998). The relationship among microhabitat characteristics, recruitment and adult abundance in the stoplight parrotfish, Sparisoma viridae, at three spatial scales. Bull. Mar. Sci., 62: 253-268.

Wilkinson, C. R. (1999). Global and local threats to coral reef functioning and existence: review and predictions. Mar. Freshwater Res., 50: 867-878.

Wilkinson, C. R. (2004). Status of coral reefs of the world. Australian Institute of Marine Science, Townsville, $316 \mathrm{pp}$.

Williams D. McB. (1982). Patterns in the distribution of fish communities across the central Great Barrier Reef. Coral Reefs, 1: 35-43.

Williams, D. McB. (1991). Patterns and processes in the distribution of coral reef fishes. In: Sale, P.F. (ed.), The ecology of fishes on coral reefs. Academic Press, San Diego, pp 437-474.

Williams, D. McB. and Sale, P.F. (1981). Spatial and temporal patterns of recruitment of juvenile coral reef fishes to coral habitats within One Tree Lagon, Great Barrier Reef. Mar. Biol., 65: 245-253.

Williams, D. McB. and Hatcher, A.I. (1983). Structure of fish communities on outer slopes of inshore, mid-shelf and outer shelf reefs of the Great Barrier Reef. Mar. Ecol. Prog. Ser., 10: 239-250. 